\title{
Use of Laryngeal Mask with Propofol Target Control Infusion for Anesthetic Management of a Patient with Escobar Syndrome
}

\author{
Olaru $\mathrm{A}^{1 *}$, Kachko $\mathrm{L}^{1}$, Hod $\mathbf{R}^{2}$ and Katz $\mathrm{J}^{1}$ \\ ${ }^{1}$ Department of Anesthesiology and Surgery Suite, Schneider Children's Medical Center of Israel, Israel \\ ${ }^{2}$ Department of ENT surgery, Schneider Children's Medical Center of Israel, Israel
}

Submission: April 12, 2018; Published: April1 24, 2018

*Corresponding author: Andrei Olaru, Department of Anesthesiology and Surgery Suite, Schneider Children's Medical Center of Israel, Israel, Tel: +972584019040; Email: herrolaru@gmail.com

\begin{abstract}
We present the anesthetic management of a 10-year-old boy with Escobar syndrome scheduled for left tympanoplasty. This syndrome may pose many problems to anesthesiologist especially regarding management of airways, difficult venous access, psychological problems because of multiples surgeries and probably association with malignant hyperthermia. Considering all of this we propose the use of LMA and TIVA for suitable operations in children with Escobar syndrome.
\end{abstract}

Keywords: Escobar syndrome; Anesthesia; Propofol; Laryngeal mask

Abbreviations: LMA: Laryngeal Mask; TCI: Target Control Infusion; TIVA: Total Intravenous Anesthesia

\section{Case Report}

Escobar syndrome or multiple pterygium syndrome is a rare autosomal recessive disease characterized by webbing of the neck, elbows, and/or knee, and joints contractures that are usually progressive and can limit mobility $[1,2]$. In literature, there are several cases describing the anesthetic management of these patients [2-5]. We present case report of 10 years old boy, $22 \mathrm{~kg}, 150 \mathrm{~cm}$ height, who was scheduled for left tympanoplasty. He was born at term, with normal Apgar score, weight of 2750g. After birth, he was treated with adenosine and beta blocker. Due to the dysmorphic features and contractures he was referred to genetic examination and Escobar syndrome was diagnosed.

At the age of 14 months, the patient underwent an uneventful laparoscopic orchiopexy under general anesthesia in another hospital without complications according to parents' report. There were no medical records available. Orthopedic follow up revealed that he started to ambulate at the age of 1,5 year and had a very unstable gait. At preoperative assessment, the physical examination revealed short stature $(150 \mathrm{~cm})$, dysmorphic features (down slating palpebral fissures, low set ears), micrognatia with a good mouth opening, (Mallampati 1), web neck, contractures of the fingers, in the wrist, hamstrings contractions, duck-like walking (on tip toes), spinal lumbar scoliosis, Grower's sign negative.
The patient was pre-medicated with midazolam $5 \mathrm{mg}$ orally. Following placement of routine monitors, a $22 \mathrm{G}$ iv cannula was inserted and anesthesia was induced with propofol target control infusion and fentanyl $50 \mathrm{mcg}$. After achieving a target concentration of $4 \mathrm{mcg} / \mathrm{ml}$ we placed $2 \frac{1}{2}$ laryngeal mask (LMA) without difficulty and mechanical ventilation in pressure support mode was initiated. During the surgical intervention, the patient was hemodynamically stable with a blood pressure around 100/60 mmHg, HR of $90 \mathrm{bpm}$, etCO2 $36 \mathrm{mmHg}$. At the end of the procedure the infusion of propofol was stopped, the LMA removed without problems and the patient was transferred to recovery room. Two hours later he was discharged home. Operation was performed by an experienced surgeon who was aware not to move the head extensively and because the head was not visible he agreed with the use of LMA.

Escobar syndrome is a rare but challenging pathology for anesthetist. Among other it involves the management of pediatric difficult airways [6]. Children with Escobar syndrome have contractures of the joints, deformities of the spine and cervical fusion, which makes endotracheal intubation a real challenge especially in the context where children are less compliant to awake intubation [3,7-9]. Worth mentioning, that good mouth opening and Mallampati 1 are not good predictors of 
successful intubation in those patients [10]. Several case reports described the use of intubating LMA, Shikami optical stylet or video laryngoscopy $[11,12]$. We decided to use LMA from the beginning. There is no consensus regarding the risk of malignant hyperthermia in patients with Escobar syndrome. Although Mayhew concluded that most patients may safely receive volatile anesthesia we decided to use propofol drip in our patient to be on the safe side.

\section{Conclusion}

In conclusion patients with Escobar syndrome may pose many problems to anesthesiologist especially regarding management of airways, difficult venous access, psychological problems because of multiples surgeries and probably association with malignant hyperthermia. Keeping in mind all the above-mentioned problems, we propose the use of LMA and TIVA-TCI propofol for suitable operations in children with Escobar syndrome.

\section{Conflict of Interest}

There is no conflict of interest or financial interest.

\section{References}

1. Thompson EM, D Donnai, M Baraitser, CM Hall, ME Pembrey, et al (1987) Multiple Pterygium Syndrome: Evolution of the Phenotype. J Med Genet 24(12): 733-749.

2. Goh, A, KW Lim, V Rajalingam (1994) Multiple Pterygium Syndrome (Escobar Syndrome)--a Case Report. Singapore Med J 35 (2): 208-210.
3. Kuzma PJ, MD Calkins, MD Kline, SM Karan, MD Matson (1996) The Anesthetic Management of Patients with Multiple Pterygium Syndrome. Anesthesia and Analgesia 83(2): 430-432.

4. Kachko, Ludmyla, Cari Maria Platis, Osnat Konen, Elchanan Bar On, et al. (2006) Lumbar Epidural Anesthesia for the Child with Escobar Syndrome. Paediatr Anaesth 16(6): 700-702.

5. Arpaci, Ayse Hande, Fusun Bozkirli, Onur Konuk (2011) Anesthesic Management for Escobar Syndrome: Case Report 2011(2): 10-13.

6. Mathew Shaji, Souvik Chaudhuri, Arun Kumar, Tim Thomas Joseph (2013) Airway Management in Escobar Syndrome: A Formidable Challenge. Indian J Anaesth 57(6): 603-605.

7. Mayhew, James F, George Mychaskiw (2009) Escobar Syndrome: Is This Child Prone to Malignant Hyperthermia? Paediatr Anaesth 19(1): 69-70.

8. Saif Ur Rehman Siddiqui, M Patti J Kymer, James F Mayhew (2004) Escobar Syndrome. Paediatric Anaesthesia 14(9): 799-800.

9. Sethi P, PK Bhatia, N Gupta, K Singh (2016) Multiple Pterygium Syndrome: Challenge for Anesthesiologist. Saudi Journal of Anaesthesia 10(3): 350-352.

10. Fang, Bingshuang, James Norris (2004) Class Zero Airway and Laryngoscopy. Anesthesia and Analgesia 98(3): 870

11. Jansen AH, Graham Johnston (2008) The Shikani Optical Stylet: A Useful Adjunct to Airway Management in a Neonate with Popliteal Pterygium Syndrome. Paediatric Anaesthesia 18(2): 188-190.

12. Xue Fu Shan, Ming Tian, Xu Liao, Ya C Xu (2009) Safe and Successful Intubation Using the GlideScope Videolaryngoscope in Children with Craniofacial Anomalies. Plastic and Reconstructive Surgery 123(3): $1127-1129$ 